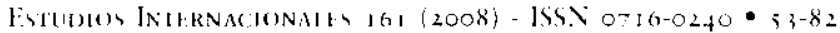

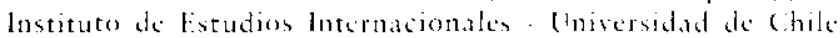

\title{
Políticas de unificación de Corea y alianzas regionales*
}

\section{Korean unification policies and regional alliances}

\author{
$\square$ \\ Martín Pérez Le-Fort \\ Alejandra Chacón Morales*
}

\section{Resumen}

Fiste articulo, como primer decumento resultante del proyecto "Korcan Unification: Historical outline of P'resumptions, Perceptions and Policies", sintetiza d debate acaldemico internacional respecto de las politicias de unificacion niacional de Corta del Sur y su relacion con el papel de Corea del Nortey los actores regionales (Rusia, (hina y lapon) y extra

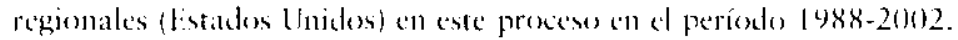
fixpone un precesamiente mu parcial de la informacion cllalitativa acumulada mediante entrevistan semiestructuradas al expertes coreanow en Corea del Sur, yue se desirrollará en otro trabajo. Como hipótesis gencral, se establece yue los intereses estratigicos de Rusia, China,

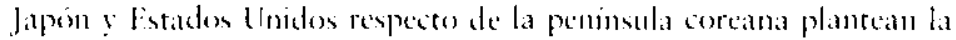
necesidad de su c'stabilizacion de acucrdo con sus propias percepciones de la seguridal, que no apuntan a apoyar un proceso de unificición

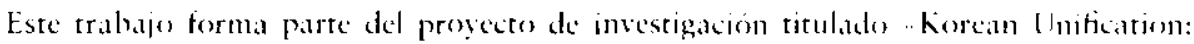

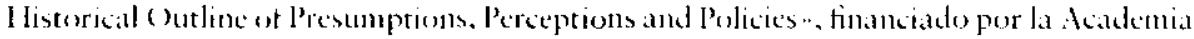

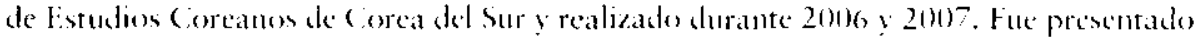

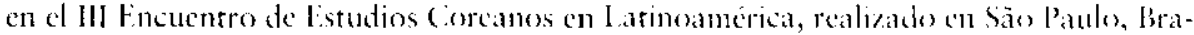
sil. los días 29 y 30 de octubre de 2007 .

Académico del lnstituto de Estudion loternacionales.

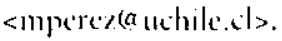

Coinvestigadora del proyecto.

Reciliclo el 27 de agosto de 2008 , aceptado el 30 de octubre de 2008. 
naciomal coreano, sino por d comerario a mantener el stitu yuo, lo yue secontradice com los intereses surcoreanos al respecto.

Palabras ciavl: Corca, unificición naciomal, equilibrios estratégicos, seguridad regiomil.

Abstract

lhis first document restlting from the project on " Korean Inification: Historical (Outline of l'resumptions, lerceptions and Policies" is a whthesis of the international academic dehate on national unitication policies in South korea and their relationship with the role played in the process by North Korea and regional (Russia. China and Japant and extrarregional actors (Inited States) in 1988-2002. It includes a very partial processing of cualitative information gathered by means of semi-formal interviews to korean experts, which will be developed in a second document. As an overall hypothesis, it contends that strategic interests of Russia, (hina, Japan and the United States regarding the korean peninsula point to the need of its stabilization, according to their own perceptions of security that do not imply supporting a Korean process of national unification but rather maintaining the status yuo, which conflicts with south Koreas interests.

Key worms: Korea, national unifiation, strategic balance, regional security: 


\section{INTRODUCCIÓN}

Este artículo es la primera parte de la presentación de los resultados de la investigación titulada "Korean Unification: Historical Outline of Presumptions, Perceptions and Policies", desarrollada en 2006 y 2007 , con el financiamiento de la Academia de Estudios Corcanos y que incluyó trabajo de campo en Corea del Sur durante el mes de julio de 2006. Para llevarla a cabo, se utilizaron principalmente entrevistas semi-estructuradas a los principales expertos de Corea del Sur sobre temas de unificación nacional. El grupo incluyó expertos del mundo académico y del gobierno.

Fn este marco, cl documento apunta a establecer el estado del debate académico internacional respecto de las políticas de unificación nacional en el periodo antes mencionado, orientado principalmente al análisis bibliográfico. A lo anterior se agrega un procesamiento muy parcial de la información cualitativa acumulada, la que será objeto de un desarrollo más amplio en un segundo artículo. El estudio permitió comprobar que la percepción de los expertos surcoreanos sobre a estos tópicos difiere mucho de la visión comúnmente accptada por el medio académico internacional, en especial de las fuentes estadounidenses, y que en clla influye también de manera significativa el debate político interno de Corea del Sur.

Como hipótesis general, se postula que los intereses estratégicos de Rusia,
China, Japón y Estados Unidos en la península coreana plantean la necesidad de su estabilización de acuerdo con sus propias percepciones de la seguridad, pero no apuntan a apoyar un proceso de unificación nacional coreano, sino a mantener el statu quo, lo que se contradice con los intereses surcoreanos al respecto.

\section{ANTECFDFNTES históricos}

Descle que surgió el primer reino de Chosón (del chino "chao", mañana, y "xian", fresca o calma), generalmente traducido como "país de la calma matutina", Corea fue habitada por pueblos bárbaros del este (Dongyi en chino)'. Si bien la visión mítica de Corea remonta su creación al año 2333 A.C. por Tan'gun (Seth, 2006: 16), como una verdadera entidad política, solamente se tienen referencias al respecto desde el año 109 A.C., cuando fue atacada y conquistada por los chinos.

Durante la dinastía Han, China logró controlar la parte norte de Corea tras atacarla los años 109-108 A.C. y dominó Chosón durante cuatro siglos. Los chinos establecieron cuatrocomandancias militares en la región ${ }^{2}$, algunas de las cuales fueron abandonadas grad-

Así como en chino Japón sc denomina "rithen", u origen del sol (Seth, . lichacl J., A Concise History of Koreal I amham: Rowman \& littleticlds l'ublishers loc., 2006. p. 17).

Las comandancias no eran colonias, sino parte integral del territorio chino. 
ualmente ofusionadas con otras, pero constituyeron un primer paso hacia un contacto cultural más profundo (Lee, 1984: 19). Las relaciones con China no fueron fáciles y la región llegó a ser un lugar de exilio de disidentes chinos, lo que dio inicio a un proceso de contacto cultural con ellos. Este fue el comienzo de un proceso de sinificación de Corca, que fue muy importante para la posterior identidad coreana.

Koguryo se constituyó como un reino en el norte de la península coreana y Manchuria. Su creación evitó el contacto directo de grupos tribales sureños coreanos con China. Al parecer, la parte meridional de Corea, militar e institucionalmente mas débil, estaba poblada por las tres tribus Han (Samban) establecidas al sur del rio Han (Manhan, Chinhan y Pyonhan) (Nahm, 1993:21). Al sur de ellos habitaba otro pueblo, probablemente $\mathrm{Wa}_{\mathrm{a}}$ en la isla de Cheju, al que no podria denominarse estrictamente japonés porque estaba formado por tribus que habitaban distintas zonas de Japón occidental y de ambos lados del estrecho que separa a Japón de Corea (Tsushima) (Seth, 2006: 23). Los coreanos de sur llaman oficialmente a la nación coreana Han'kuk (país de los Hanl).

Con el gatillante del conflicto entre los emergentes reinos de Koguryo, Paekche y Silla, surgidos de las tribus Han y la búsqueda china de mayor influcncia, este áltimo se convierte en reino unificador de casi toda la península coreana. Si bien los reinos conquistados por Silla no constituáan originalmente una unidad étnica, esta se fue creando con el tiempo, absorbiendo también la idea china de gobierno, religión, ética, arte, música, familia, estructura y moda, que se combinó con las tradiciones y el desarrollo indigenas (Scth, 2006: 34-39). Por otra parte, con diversos nombres este reino aumentó su población y su riqueza y amplió sus fronteras hasta alcanzar, a mediados del siglo XV, su actual extensión.

Tras la decadencia del reino Silla y la posterior reunificación bajo Koryo, se inician nuevos procesos dinásticos. Desde 935 hasta 1392 el país fue controlado por la dinastía Wang, que estableció la capital en Kaesong y cambió el nombre del país a Koryo (Lee, 1984: 100), autopercibido como sucesor de Koguryo en su lucha con Silla. Koryo imitó en gran medida las estructuras administrativas de la dinastía china Tang (Seth, 2006: 79). Luego, entre 1292 y 1910 , Corea fue gobernada por la dinastía Yi, que le devolvió al país el nombre de Chosón y que gobernó desde Seúl (Seth, 2006: 79).

Este largo proceso cstuvo cruzado por los procesos de atomización y reunificación chinos, por la invasión mongola, por los primeros ataques organizados de los japoneses y por la invasión de los Jurchen, que dio pie a la uiltima dinastía china de los Qing.

Fl contacto inicial con los chinos y el aislamiento relativo dieron lugar a que surgiera una identidad coreana propia. Si bien la influencia cultural china se mantuvo mediante el budismo y el confucionismo chinos, la identidad 
coreana, fortalecida, logró importarles sus particularidades. Por otra parte, esta identidad se acrecentó por la presión militar, primero china y luego japonesa, a partir del siglo XVI, después de que Japón logró su propia unificación durante el shogunato Tokugawa.

Lo anterior explica el contacto inicial con dos poderes regionales cuya influencia sobre la península se mantendría hasta el siglo XIX, cuando se les sumarán Rusia en busca de puertos de aguas cálidas y las potencias occidentales, aunque aún muy concentrados en China. Ya en la primera mitad del siglo XX, una Corea debilitada, junto con una China imperial bajo la abierta decadencia de la dinastía Qing, generan las condiciones para que Corea se transforme en colonia japonesa desde 1910 hasta 1945 (Lee, 1984: 313 ).

Luego de la segunda guerra mundial, por iniciativa estadounidense ambas Coreas se dividen en el paralelo $38^{\circ}$ (Kim, 2006: 237). Con el inicio la guerra de Corea, generada por un fuerte error de cálculo de Kim Il-sung, lider de Corea del Norte, un reticente apoyo soviético y la búsqueda de un rol internacional preponderante por parte de la recién creada República Popular China (Kim, 2006: 50-51), se definió en gran medida el sistema de relaciones regionales hasta fines de la guerra fría.

A partir de la guerra fría las dos superpotencias, la Unión Soviética y los Estados Unidos, concentraron una intensa presión sobre un punto focal: la península de Corea. En 1945 estadouni- denses y rusos la dividieron, situación que se hizo permanente a fines de esa década. Después de la guerra de Corea, que dejó al país en ruinas, comenzó a desarrollarse una de las zonas más militarizadas del mundo.

Cabe recordar que en 1949, cuando Corca del Norte planeó invadir Corea del Sur, le planteó a Stalin la necesidad de apoyo, pero este lo rechazó. Al año siguiente, sin embargo, al cambiar el escenario internacional, estuvo dispuesto a darlo. Las razones de ello fueron el desarrollo de la bomba atómica rusa, la retirada estadounidense de Corea del Sur y la exclusión de este áltimo dentro del perímetro defensivo de Estados Unidos (Kim, 2006: 114).

\section{LAS POLÍTICAS DE UNIIICACIÓN NACIONAL EN COREA}

\subsection{Las relaciones intercoreanas}

Desde 1945, y más marcadamente tras el armisticio de la guerra de Corea, las relaciones intercoreanas se han caracterizado por una serie de altibajos. Ambas partes se han acusado de poner obstáculos para evitar que las conversaciones fructifiquen (Castillo, 2002: 166).

Entre estas acusaciones, cabe mencionar las maniobras militares conjuntas de Corea del Sur y Fstados Unidos $y$ algunos incidentes tales como el atentado de 1983 en Rangún, Myanmar, en que murieron 21 surcoreanos, entre ellos cuatro ministros (Castillo, 2002: 
166), y que Corea del Sur atribuyó a agentes norcoreanos.

Sin embargo, el diálogo avanzó de manera significativa a partir de 1991 mediante la firma del citado "Acuerdo de Reconciliación, no Agresión, Intercambio y Cooperación entre el Norte y el Sur", conocido también como "Acuerdo Básico" entre ambas Coreas. Con todo, este se vio interrumpido con e] fallecimiento del líder norcoreano Kim Il-sung y no puede hablarse de un avance hasta la primera Cumbre Intercoreana celebrada en 2000 , en la cual se tocaron principalmente temas huminitarios como las visitas entre las familias separadas y la ayuda a Corea del Norte, así como también el tema de la reconciliación nacional.

La mayoria de los analistas coincide en que los acuerdos de esa cumbre favorecieron mayoritariamente a Corea del Norte y en que muchos de ellos fueron difíciles de cumplir debido a los vaivenes de las políticas de Kim Jong-il. Sin embargo, siete años después de la primera Cumbre y ad portas de la elección de diciembre de 2007 se celebró una segunda Cumbre, nuevamente en Pronyang, esta vez con el presidente Koh Moo-hyun y el líder Kim Jong-il. La diferencia entre ambos encuentros tiene varios simbolismos: el presidente Roh pasó a pie la línea divisoria entre las dos Coreas en señal de acercamiento y la prensa internacional pudo informar sobre los pormenores de la Cumbre desde la misma Pyonyang.

Los acuerdos de la segunda Cumbre fueron más completos que los de la an- terior. Los más relevantes se refieren a la búsqueda de firmar la paz, es decir, a dejar de lacto el armisticio de la guerra de Corea y alcanzar una paz definitiva, al establecimiento de una zona especial en el mar Amarillo para evitar incidentes y al tema nuclear, además de los que ya figuraban en el diálogo. Ante la promesa de Corea del Norte de poner fin al programa nuclear antes de diciembre de 2007 , formulada en el diálogo de las "Seis Partes", ambas Coreas han declarado su voluntad de trabajar conjuntamente en este tema?

Sin embargo, las elecciones de diciembre de 2007 en Corea del Sur alteraron profundamente la viabilidad de compromisos asumidos en los acuerdos, ya que a raíz del triunfo de la oposición hay pocas expectativas de que el nuevo gobierno esté dispuesto a respetarlos, debido a que tiene una postura más crítica frente a Corea del Norte.

\subsection{Politicas de unificación}

Por más de dos décadas tras el establecimiento de la República de Corea, Corea del Sur negó la existencia de dos Coreas, planteándose como gobierno legítimo de toda la península (Levin y Han, 2002: 5). El tema de la unificación comenzó a abordarse en la década de los setenta por el presidente Park Chung-hee. En el discurso pronunciado con motivo del $25^{\circ}$ aniversario de la li-

Fucnte: http://spanish.korea.nct. 
beración de corea del dominio japonés (1.5 de agosto de 1970), Park sugirió la idea de una coexistencia pacífica, para reemplazar la lógica de confrontación militar por la de cooperación socioeconómica (levin y Han, 2002: 61. FI Comunicado Conjunto de 1972, yute reflejaloa un dialogo informal previo (de carácter secreto) y que inicialmente estuvo en manos de los servicios de inteligencia surcoreanos), da comicnzo a los intentos de unificación (Levin y Han, 2002: 6).

La polírica de Park Chung-hee tomó forma en la "Declaración de Política Exterior Especial Respecto de Pazy Unificación" a partir de 1973 y redujo la oposicion surcoreana al ingreso simultáneo de ambas Coreas a las . Vaciones Unidas (Levin y Han, 2002: 7).

En su "Declaración de la Politica del Año Nuevo de 1982", el presidente Chun Doo-hwan anunció una nucva fórmula de unificación pacífica en reemplazo de las relaciones antinaturales entre ambas Coreas, que contemplaba el establecimiento de relaciones completamente normales y promovía un amplio rango de medidas de intercambio y cooperación en el campo del transporte, las comunicaciones y otros (Levin y Han, 20()2: 7).

Roh Tae-woo, en su "Declaración Presidencial Especial" de 1988, insistió en cue la división no era naturaly en que ambas Coreas no debian verse como adversarias, procurando alcanzar una asociación mutua para lograr la prosperidad común. Propuso medidas de intercambio recíproco, comercio abierto y cooperación para desarrollar una comunidad nacional común (Levin y Han, 2002: 8). Además, establecio el Comite para la Promoción de la Cooperación y el Intercambio lntercoreano y planteó la idea de un desarrollo equilibrado de ambas Coreas, to que implicaloa una ceventual asistencia a Corea del Norte (Levin y Han, 2002: 8). Por otra parte, sostuvo que era preciso prestar apoyo para mejorar las relaciones norcoreanas con Estados Unidos y Japón.

I. Declaracion de Roh Tae-woo contemplaba un acercamiento gradual de acuerdo con el cual se realizaría una serie de actividades propias de los Fstados-nación independientes bajo la rúbrica de "coexistencia pacifica", que no implicaba una integración formal. La Declaración se vio reforzada por la Nordpolitik de Roh hacia los países comunistas, que tuvo una respuesta positiva. Fn 1991, durante su gobierno, se llegó al citado "Acuerdo de Reconciliación, no Agresión, Intercambio y Cooperación entre el Vorte y el Sur" entre ambas Coreas (Levin y Han, 2002: 9).

Fl acuerdo incluyó amplios compromisos, entre los cuales destaciaba el respeto por los asuntos internos de cada uno. Se promovieron diversos mecanismos para la realización de visitis de familia, el intercambio deportivo, la cooperación en temas culturales la creacion de oficinas especiales para ello. Todo ello se complemento con la Declaración Conjunta de Desnuclearización de la Península Coreana. Pero la 
muerte de Kim Il-sung, en 1994, detuvo temporalmente el proceso (1.evin y Han, 2002: 9-10).

Fl sucesor de Roh, Kim Young-sam, adhirió a los componentes clave de este proceso, reforzándolo con declaraciones puiblicas de que Corea del Sur no pretendia una unificacion por absorciom, esto es, al estilo de la unificación alemana (Levin y Han, 2002: 9).

El retiro de Corea del Norte del Tratado de No Proliferación Nuclear afectó la política de Kim, ya que Estados Unidos domino el tema del diálogo con Corea del Norte. Por otra parte, los sectores conservadores surcoreanos limitaron su politica, lo que sumado a la muerte de Kim Il-sung en Corea del Vorte, afectó las posibilidades de diálogo.

Ios lineamientos esbozados por cl presidente Kim Dae-jung en 1998 incluirán los siguientes tres principios básicos (Kim, 1998):

a) No se tolerarán provocaciones armadas de Corea def Norte,

b) No se intentará una toma del poder o absorcion de Corea del Nortc, $y$

c) Se ampliarán la reconciliación y la cooperación.

A partir de estos lineamientos, se inició una nueva serie de actividades para fomentar reuniones e intercambios entre familias separadas por la gucra de Corea. De acuerdo con ello, Corea del Sur aplicó una política llamada de compromiso o "sunshine policy" hacia el norte. Sin embargo, Corea del Norte ha adoptado un criterio estratégion fundamental al respecto, consistente en el fin de la presencia estadounidense en Corea del Sur. Después de la Cumbre Intercoreana de junio de 2000, hubo frecuentes intercambios entre ambas Coreas, pero en 2001 los frutos de esta política no eran claros, en especial en lo que se refiere a la tensión militar. La declaración conjunta Norte-Sur suscrita cl año 2000 habia puesto énfasis en la unificación, los temas humanitarios, la cooperación social y economica y el intercambio y la cooperación internacionales (Levin y Han, 2002: 27-29).

Desde noviembre de 2001, a fin de preservar su "sunshine policy", Corea del Sur trató de reanudar el diálogo intercoreano suspendido por Corea del Vorte. La idea era recahar la cooperacion y el apoyo de los Fstados Unidos (su aliado), así como de Japón, China y Rusia.

Cabe recordar que al ganar la clección presidencial Roh Moo-hyun insistio en que, pese a las ambiciones nucleares de Corea del Norte, la mejor forma de avanzar era continuar y quizás fortalecer la anterior politica de compromiso.

\section{¿NULVA POSTURA IDEL GOBILRNO DE COREA DEl Sur?}

El presidente Roh Moo-hyun reemplazó de la "sunshine policy" por la "peace and prosperity policy", orientada hacia una paz duradera. En esta se 
planteó que no se toleraria el desarrollo nuclear y que se incentivarian el intercambio de personas y la cooperacion conómica. Al respecto, cabe tener presente el impacto económico de la tensión sobre Corea de Sur. Por otra parte, para los sectores jóvenes, base política del presidente anterior, Roh Moo-hyun, los norcoreanos son como hermanos, no enemigos, lo que refleja una fuerte percepcion de identidad, más allá de la politización interna del tema.

Dentro de las politicas hacia Corea del Norte implementadas durante el gobierno surcoreano anterior, cabe mencionar el intercambio de visitas de familias separadas, las medidas relacionadas con fertilizantes y alimentos, cl término de la conexión ferroviaria Seul-Sinuiju y otras, además de medidas simbólicas como la eliminación de la propaganda en la zona desmilitarizada. Sin embargo, no han dejado de ocurrir incidentes esporádicos, por ejemplo, navales.

En las contrevistas realizadas en Seúl, los expertos mencionaron el cambio en la percepción surcoreanla sobre Corea del Norte, esto es, que se trata de "hermanos" y no de enemigos. Además, hubo renuencia a referirse a los temas militares dehido a cue ello podria incomodar a los Estados Unidos. En general, la impresión e's que el proceso de unificacion es de largo plazo y que en el ha desempeñado un importante papel la "generacion 386". Desde el punto de vista más critico y conservador propio del actuil gobierno, los entrevistados opinaron que la política surcoreana hacia Corca del Norte había legitimado el régimen norcoreano y le habia permitido sobrevivir.

\subsection{Recordande les escenarios de unificacion}

Muchos especialistas han especulado respecto de posibles escenarios de unificación coreana. Sin embargo, en general las alternativas serian de unificación por integración pacifica, unificación por colapso y unificación por guerra (Pollack, 1999: 45). Otros concehirian dos amplias alternativas: por una parte, aterrizaje suave, un escenario en que lo fundanental es que Corea se reunificará por acuerdo entre el Norte y el Sur y con que está implicito que la reunificacion será resultado de negociaciones pragmáticas motivadas por el deseo común de poner fin a la división artificial de la nación y, por otra, aterrizaje difícil, en que la unificacion será resultado del colapso del régimen de Corca del Norte o del Estado norcoreano debido a su absorción por Corea del Sur (Pollack, 1999: 39).

Las proyeciones de los costos de la reunificación fluctúan entre los 290 mil millones de dólares (estimación realizada en 1994 para un período de 32 años) $y$ los 3,2 billones (estimación efectuada en 1997 para un periodo de 10 anos) (Wolf y Akramov, 2005: 17-18). 
2.4 Existencial de tm conse'nso intermo sobre las politicas de unificación nacional

Si bien hay consenso respecto de la necesidad de un proceso de unificación a largo plazo, los mecanismos para alcanzarla varían profundamente. L.o que se vio en los intentos realizados en el período de gobierno autoritario de Corea del Sur fue una suerte de utilización política de esta aspiración por las dictaduras militares, a fin de alcanzar un impacto position en la opinión pública. Sin embargo, ello dio lugar a un cambio en la percepción acerca de la necesidad de unificación en amplios sectores sociales y politicos. Con la llegada de la democracia, en Corea del Sur se planteó una política más agresiva en torno a la unificación, en especial a partir de Kim Dae-jung, al parecer desconociendo importantes esfuerzos anteriores como los de Roh Tae-woo.

En cierta medida, las políticas de unificación nacional se han ido politizando gradualmente y han roto la unidad de la opinión pública respecto del tema. Fin esta dicotomía, encontramos por una parte intentos por hacer gestos simbólicos hacia Corea del Norte, una husqueda de apoyo al desarrollo economico y asistencia humanitaria y, por la otra, un fuerte escepticismo en relación con estos gestos de buena voluntad que no tienen respuesta satisfactoria de la contraparte norcoreana. Los sectores del anterior gobierno surcoreano veían una política coherente y de largo pla- zo y percibian muy negativamente la injerencia de terceros, como Fstados Unidos, que seguían vinculando su apoyo a las dictaduras militares. Por otra parte, en el nuevo gobierno se percibe que ya no es viable seguir ayudando a un régimen que da claras muestras de ser una amenaza real y permanente, con sus prucbas misilísticas y un programa nuclear aún no desmantelado y, sobre todo, yue no cumple sus compromisos. Desde este punto de vista, sería ingenuo llegar a un compromiso con Corea del Norte.

En este contexto, difícilmente puede hablarse de consenso interno respecto de las políticas de unificación, especialmente cuando se las utiliza como herramienta electoral. Fon síntesis, la sociedad coreana concuerda en la necesidad de la unificación nacional, pero no respecto de los mecanismos para alcanzarla.

Fn las entrevistas realizadas en Seuil, las respuestas de los expertos fueron muy variadas. En primer lugar, ofrecieron una visión bipolar respecto del apoyo a las políticas de unificación nacional. Los partidos de derecha, que ganaron las uiltimas elecciones, tuvieron una visión crítica, mientras que la de quienes respaldaron al gobierno del presidente Roh fue muy positiva. Fl sector de negocios mostró poco interés y entre los medios de comunicación, los de corte conservador fueron críticosy los progubernamentales las apoyaron. La opinión publica estuvo dividida

Resumen de las entrevistas a expertos en Corca del Sur. 
pero muy interesada en la unificación (la pregunta fue cuándo y qué tipo de unificación) y, por su parte, las Fuerzas Armadas fueron críticas. Los diferentes grupos etarios percibian la unificación de manera distinta: los mayores son más anticomunistas y a los más jóvenes les interesa menos el proceso, pero les preocupan mucho sus eventuales costos económicos.

\subsection{Viabilidad del régimen norcoreano}

Después de 1945, la península coreana comienza a apuntar hacia su propio desarrollo económico mediante dos opciones, socialista y capitalista, que tuvieron distintos resultados. Corea del Norte logró éxitos económicos en los años sesenta, pero la política de "Juche" (o autosuficiencia) implementada por el dictador Kim Il-sung condujo al estancamiento. Esto no es ajeno a lo sucedido en China y al impacto en el lider norcoreano de la revolución cultural china. Si bien aceptó ayuda de la Unión Soviética, en general tendió a aislarse del mundo, en el cual volvía a manifestarse el viejo debate de la tradición marxista entre la visión más determinista de la Unión Soviética y la de carácter más voluntarista de China. Una de las ideas de la "Juche" era encontrar un punto medio entre la Unión Soviética y China, lo que evidentemente ponía de manifiesto una importante cuota de nacionalismo. A pesar del discurso sobre la "Juche", Corea del Norte "ha dependido de la asistencia externa a través de toda su historia" (Nolind, 2004: 20).

La crisis del modelo Juche de desarrollo (autoconfianza o autodependencia) se inició en los años setenta, con Kim Il-sung. La economía se había organizado en torno a compañias estatales y cooperativas (estas últimas con derechos de uso). Fn respuesta a los problemas económicos, en el periodo 1970-1973 se intensificó "el grado de centralización de la planificación agrícola” (Noland, 2004: 19). Al respecto, en las zonas rurales se impuso un estilo chino de "revolución cultural" (Noland, 2004: 20). El proceso de planificación no fue eficaz, especialmente en el sector rural, y si a ello se agrega que en los años setenta las instancias crediticias internacionales congelaron la ayuda a Corea del Norte (Noland, 2004: 20) y que en los años ochenta se redujo la asistencia soviética, en 1987 cl flujo de recursos externos se tornó negativo (Noland, 2004: 20).

La economía formal de Corea del Norte comprende empresas estatales y colectivas agrícolas; es un sistema centralmente planificado y controlado monocráticamente (Lee y Yoon, 2004: 46-47). Además, existirían otras economias: la economía militar o segunda economía (independiente y que maneja la producción de bienes militares) y una economía "privada" obviamente informal, por ejemplo, en el ámbito agrícola (Lee y Yoon, 2004: 48-49). La economia formal se ha debilitado muchísimo debido al colapso de las empresas estatales, mientras que la economía 
informal "privada" ha crecido gracias a los esfuerzos de la gente por sohrevivir (Lee y Yoon, 2004: 59). Por su parte, la economía militar ha experimentado una pequeña contracción (Lee y Yoon, 2004: 59).

Determinar el tamaño de la economía de Corea del Norte es muy difícil debido a que, al igual que en la Unión Sovićtica, se excluyen los "servicios intermedios" (Wolf y Akramov, 2005: 9-10). Fn 2002, el PIB fue de 22.800 millones de dólares, en comparación con 727.800 millones en Corea del Sur (Wolf y Akramov, 2005: 11). El crecimiento acumulado entre 1990 y 2002 fue de $-22,2 \%$ frente al $81 \%$ de Corea del Sur (Wolf y Akramov, 2005: 12).

Desde una perspectiva "colapsista", definiendo colapso como la interrupción de la división del trabajo en la economía nacional, en los años noventa Corea del Norte experimento un colapso económico, ya que su conomía fuc incapaz de producir los bienes necesarios para mantener la división del trabajo (Eberstadt, 2004: 65). Sin embargo, la situación varió a mediados de la presente década y a partir de 1998 el país registra una tasa más elevadia de crecimiento. La pregunta que cabe plantearse es cómo explicar esta mejora: efecto de la ayuda surcoreana, ayuda occidental, actividades ilegales, venta de armas u otros. Esto no es muy claro, perocontra todos los pronósticos Corea del Norte está siendo capaz de sobrevivir.

\subsection{El interés norcoreano por un} proceso de unificación pacifica y gradual

A Corea del Norte le ha interesado la unificación de la península desde la división del país. La guerra de Corea fue un esfuerzo extremadamente arriesgado por alcanzarla y casi derivó en la destrucción del propio régimen norcoreano de no mediar la intervención de sus aliados. F. dilema que se presenta aquí es que ha querido la unificación en sus propios términos y que en el curso del proceso ha experimentado un deterioro económico.

Surge la duda de si la participación de Corea del Norte en el diálogo obedece a una flexibilización de su política, a un intento por aliviar su débil situación económica o a la contención de su amenaza más sustantiva, que son los Estados Unidos. Pero este país es una amenaza que da cohesión interna y que, al mismo tiempo, en un proceso de negociación por el programa nuclear de uso militar o por otras armas de destrucción masiva puede ofrecer algunas soluciones económicas. Sin caer en el crudo realismo de la segunda alternativa, puede entenderse que también hay algo de flexibilización y de creación de nuevas identidades.

En la perspectiva norcoreana respecto de la relación Norte-Sur pueden definirse los tres principios siguientes (Armstrong, 2004: 41):

a) Corea del Norte es el verdadero representante del pueblo coreano y el 
régimen surcoreano, respaldado por un muy hostil Estados Unidos, es una grave amenaza para su existencia. Para enfrentarla, debe establecer una fuerte defensa (no solo militar, sino también ideológica).

b) E.I pueblo surcoreano, en oposición a su gobierno, daría cálida bienvenida a la unificación, pero lo afecta la propaganda estadounidense y la hostilidad del liderazgo surcoreano. Corea del Norte debería perseguir una táctica de "frente unido" para obtener apoyo en Corea del Sur y en el exterior.

c) La posición norcoreana ganará el apoyo del pueblo de ambas Coreas, porque es moralmente correcta. En ese sentido, es posible negociar con Corea del Sur y no interferir en su sistema porque, a la larga, este vería la superioridad del régimen norcoreano y lo adoptará.

En las entrevistas realizadas en Seúl, los expertos mencionaron algunos de los objetivos principales de Corea del Norte: obtener asistencia económica de Corea del Sur, usarla de mediador con los Estados Unidos y lograr mejores condiciones para la unificación. Aparentemente, no hay interés explícito por un proceso de unificación gradual y pacifica.

\section{I OS ACIORES INVOLUCRADOS}

\subsection{El rol de los Estados Unidos:} ¿por qué multilateralizar el conflicto?

A partir de la «diplomacia de las cañoneras" del siglo XIX, la relación de los Estados Unidos con Corea no ha sido fácil. Al respecto, cabe recordar el incidente del General Sherman en 1866 y la represalia de 1871 (Kim, 2006: 234). Los coreanos tenían una impresión negativa de los estadounidenses a causa de sus intentos imperialistas de mercantilismo y proselitismo y, por su parte, estos percibían a los coreanos como ermitaños y violentos ( $K$ im, 2006: 235). Los tratados suscritos entre Corea y los Estados Unidos se consideraban como una forma de contener la expansión japonesa (Kim, 2006: 235). El desinterés estadounidense generó a su vez el interés coreano de que Rusia desempeñara un papel más destacado. Sin embargo, Estados Unidos prefería a Japón antes que Rusia y miraba positivamente un predominio estadounidense en Filipinas y uno japonés en Corca (Kim, 2006: 236).

En la guerra de Corea hubo 36.940 muertos en acción, 3.737 desaparecidos, 92.134 heridos, 400.000 bajas surcoreanas y alrededor de 2 millones de bajas chinas y norcoreanas (además de innumerables bajas civiles), todo lo cual dejó una profunda huella negativa (Kim, 2006: 240).

Tras la división Corea del Sur percibió a los Estados Unidos en forma positiva y no necesariamente como 
un actor imperialista o colonialista. Ello quedó de manifiesto durante el gobiemo del presidente Rhee, que se caracterizó por una marcada dependencia económica de ese país (Cumnings, 2004: 343-349).

Fvidentemente, durante mucho tiempo y hasta fines de los años ochenta la guerra de Corea eliminó cualquier posibilidad de diálogo entre ambas Coreas, aunque en 1973 hubo un intento por realizarlo. Asimismo, en 1974 Corea del Norte ofreció al Congreso estadounidense reemplazar el armisticio por un tratado de paz. En 1975 y 1976 hubo algunas reacciones positivas al respecto en algunos sectores (Henry Kissinger). Se buscó el reconocimiento cruzado (China-Unión Soviética con Corea del Sur y Estados Unidos con Corea del Norte) (Kim, 2006: 243). Durante el gobierno de los presidentes Ford y Carter, los norcoreanos continuaron sus intentos.

En 1983 se realizaron algunos es. fuerzos a través del presidente Hosni Mubarak (Kim, 2006: 243). Estados Unidos rechazó toda propuesta que significara excluir a Corea del Sur. En 1984, Corea del Norte propuso realizar una conferencia tripartita (tratado de paz. y fin del estado de hostilidades) (Kim, 2006: 243). Posteriormente, se apuntó al retiro de las tropas estadounidenses y al inicio del diálogo intercoreano sobre la unificación, incluidos

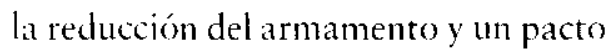
de no agresión. Sin embargo, ambos procesos se desarrollarían en forma independiente. Por su parte, Estados
Unidos quería involucrar en este diálogo a China (Kim, 2006: 243).

Al terminar la guerra fría, los gobiemos estadounidenses rechazaron estas propuestas, con algunas excepciones como la "iniciativa modesta" de diálogo de la administración Reagan, que permitía la exportación de ciertos productos y los contactos no gubernamentales (Kim, 2006: 243). Sin embargo, en el gobierno de George Bush padre, la iniciativa no prosperó (Kim, 2006: 244).

La prioridad obvia, tanto en el gobierno de Bush padre como en el posterior de Clinton, fueron al parecer las consecuencias del colapso del bloque soviético, como por ejemplo el tema de las armas nucleares. Al inicio de la administración de Clinton, Estados Unidos no dio pasos sustantivos respecto de Corea del Norte. En 1993, este anunció su retiro del Tratado de No Proliferación Nuclear (TPN) y rechazó las inspecciones del Organismo Internacional de Energía Atómica (OIEA). Las tensiones aumentaron, pero China amenazó con hacer uso del veto en el Consejo de Seguridad de las Naciones Unidas (Kim, 2006: 244).

I as negociaciones bilaterales se centraron en un acuerdo sobre el desarrollo de centrales nucleares de agua ligera, que se completarían en 2003. Estados Unidos trató de nultilateralizar el tema (China, Japón, Rusia, OIFA, Consejo de Seguridad) (Kim, 2006: 245), pero en 1994 los norcoreanos dieron un paso adelante y eliminaron el material nuclear de la central de Yongbyon. 
Para Corea del Norte, las sanciones de las Naciones Unidas conducian a una declaración de guerra. En junio de 1994 ya se discutia una solución militar (Kim, 2006: 24.5).

Los sectores moderados del gobierno estadounidense (por ejemplo, WiIliam Perry, Secretario de Defensal), no quisieron llegar a ese punto, lo que condujo al "Acuerdo Marco" de 1994, que incluyo un limitado compromiso entre ambas partes. Entre los aspectos considerados cabe mencionar la provisión de reactores de agua ligera (el primero de los cuales se completaría en 2003) y el intercambio de documentos solbe el uso pacífico de la energía nuclear, el desmantelamiento de los reactores de grafito, el congelamiento de los cue estaban en construcción y el suministro de combustible a Corea del Norte por un máximo de 500.000 toneladas anuales (Kim, 2006: 245-246).

En este contexto, Corea del Norte se comprometió a no desarrollar armas nucleares y Estados Unidos a no amenazar con usarlas en su contra. Además, incluía el compromiso de diálogo intercoreano (Kim, 2006: 246). E1 problema fue que, a la inversa de Corea del Norte, Fstados Unidos consideró que el actuerdo no cra vinculante.

Luego de la muerte de Kim Il-sung en 1994 y la llegada al poder de Kim Jong-il, hijo del anterior, la situación cambio. Al comienzo el nuevo líder mantuvo posiciones en el marco de las Fuerzas Armadas norcoreanas. Fue elegido secretario general del Partido de los Trabajadores en 1997, conservó su posición de mando en el ámbito militar y fuc proclamado presidente vitalicio (Scaliapino, 2006: 146). Cabe recordar que a fines de la década a la tradicional política de "Juche" (autoconfianza o autodependencia) se sumó la de "Songun" (lo militar primero) (Scaliapino, 2006: 146).

Fn 1995, a instancias de los Fstados Unidos, Japón y Corea del Sur crearon la Korcan Peninsula Energy Development Organization (KEl)()) para el desarrollo del programa de reactores de agua ligera antes mencionado. En los años siguientes, Corea del Norte seguirá solicitando ayuda (Scaliapino, 2006: 148).

F.n 1996, Estados Unidos propuso iniciar un diálogo de cuatro partes que incluía a las dos Coreas, China y Estados Unidos (Scalapino, 2006: 148) y que en 2003 daria lugar a las "conversaciones de las seis partes". Pe'se a que Corea del Norte prefería las relaciones bilaterales con los Fstados Unidos, las conversaciones se inciaron en 1997 (Scalapino, 2006: 149), pero se vieron profundamente afectadas por cl lanzamiento del misil Tacpong 1 ('n agosto de 1998, que cruzó el espacios aéreo japonés (Scalapino, 2006: 149).

Por otra parte, Corea del Norte se quejó de que no se estaban cumpliendo los plazos del programa de reactores de agua ligera y, además, de que Estados Unidos estaba atrasado en la provisión de combustible pesado. Fn ese contexto, amenazó con reabrir las instalaciones de Yongbyon. 
En 1998 se llegó a un nuevo acuerdo para cumplir con las entregas atrasadas de combustible y avanzar en la construcción de los reactores de agua ligera.

Ya en 1999 la administración Clinton hizo algunos intentos de acercimiento que al comienzo fueron poco fructíferos (Scalapino, 2006: 149). la política de Kim Dae-jung descle Corea del Sur (Cumbre de 2000$)$ y un intento de Corea del Norte por acercarse hacia China y Rusia generaron un contexto más favorable. La idea era dejar sin efecto algunas sanciones a cambio de la eliminación del programa nucleary de misiles (Scalapino, 2006: 150).

I.os norcoreanos querian que el presidente Clinton visitara el país, pero finalmente lo hizo la Secretaria de Fstado, Madelcine Albright, en 2000. 1)e la visita surgieron varios compromisos en el sentido de poner fin a la producioin y venta de misiles a cambio de com. pensaciones. Con todo, el proceso de verificacion requeriria de nuevas negociaciones (Scalapino, 2006: 150).

En esal reunión, el lider norcoreano mencionó que el término de la guerra fría permitia que las tropas estadouniclenses desempeñaran un rol estabilizador, declaración que resultó sorprendente (Scalapino, 2006: 1.50) y dio muestras de un mayor interés por el modelo de desarrollo tailandés o stueco que por el chino. Aparentemente, Kim Jong-il quería llegar a un acuerdo.

I a llegada de la administración de Bush hijo provocó algunos cambios, no continuidad, pese a los esfuerzos iniciales de Colin Powell (Scalapino, 2006: 151 ).

Cuando Kim Dae-jung, Presidente de Corea del Sur, visitó Washington D.C. en 2001 , el presidente norteamericano se mostró muy escéptico respecto de la "sunshine policy" surcoreana de acercamiento a Corea del Norte. En jumio de ese año, la administración Bush propuso avanzar en el tema del Acuerdo marco, el programa de misiles y política militar convencionales. Corea del Norte reacciono de manera bastante negativa a esas propuestas, que a su juicio perseguían "sofocar, aislar y destruir" su pais (Scalapino, 2006: 151).

Durante ese periodo, Corea del Norte procuró mejorar su situación internacional (Unión Furopea, China, Rusia y otros). Fn los Estados Unidos hubo pocas simpatias por las negociaciones con ese país, declarado "tstado paria" por Bush en su discurso de política exterior, impresión que aumentó tras los atentados del 11 de septiembre (Scalapino, 2006: 151 ). Fn 2002 la desconfianza se mantuvo, al voluarse Estados Unidos a su lucha contra el terrorismo, incluyendo a Corea en el "cje del mal" (Scalapino, 2006: 151), mientras Corea del Norte trataba de mejorar sus lazos internacionales y registraba una leve mejora económica.

FI Secretario de Estado Adjunto estadounidense, James Kelly, visitó Pyonyang en octubre de 2002 y en esa oportunidad se trató la violación norcoreana del Acuerdo Marco y de sus obligaciones con el Tratado de No Proliferación Niclear (Scalapino, 2006: 152). 
En 2002, ante la denuncia estadounidense de que Corea del Norte poseia un programa de uranio altamente enriquecido, este respondió que obedecía a su derecho a desarrollar armas contra la amenaza de Estados Unidos (K'im, 2006: 258). También argumentó que ante la amenaza nuclear estadounidense no solo tenía derecho a desarrollar ese tipo de armas, sino también otras, para defender su soberania y su supervivencia. A continuación vino la oferta de un tratado de no agresion y no uso de armas nucleares. Estados Unidos apuntaba al desmantelamiento total y Corea del Norte a congelar su programa nuclear, distinguiendo entre enriquecimiento de uranio y plutonio (Bechtol fr., 2006: 116), con lo cual pretendía trasladar lia responsabilidad de su realización a la amenaza estadounidense. Además, cllo le permitia ganar tiempo para concinuar su desarrollo a partir de instalaciones previamente congeladas y condicionar su renuncia al programa nuclear sobre la base de la firma de un tratado que podría representar un futuro retiro de las fuerzas estadounidenses de la península. A juicio de Estados Unidos, ello significaloa garantizar la supervivencia del régimen de Corea del Norte y podría afectar seriamente la capacidad disuasiva de Corea del Sur. Pese a ello, ambos participaron en las "conversaciones de las seis partes" (Cha y Kang, 2003: 140-144).

La Declaración de Principios del 19 de septiembre de 2005 turo un inicio optimista debido a las dificultades para llegar a un acuerdo con que se habia tropezado en diciembre de 2003. Corea del Norte habia acordado abandonar toda arma nuclear o programa al respecto, accediendo a retornar al Tratado de No l'roliferacion . Nuclear y a aceptar las salvaguardas del OIEA en una fecha proxima, lo que también implicaba aceptar las inspecciones de este organismo. Asimismo, acordó reafirmar el Acuerdo de Desnuclearizacion Corea del Norte-Corea del Sur de 1992. Dos concesiones importantes no relacionadas con cl tema nuclear fueron la de un régimen de paz coreano y la relativo al secuestro de ciudadanos japonenses.

Al parecer, Corca del Norte no renunció a la aplicación de las políticas anteriores al acuerdo (programas de armas nucleares y de enriquecimiento de uraniol.

De acuerdo con los resultados de una encuesta realizada por Research \& Research en encro de 2004 , Fstides Unidos era la principal amenaza para Corea del Sur (39\%), después de Corea del Norte (33\%). Fisto se explica en parte porque la opinión pública relaciona a ese país con el período de las dictaduras. Fn el rango de 20-30 anos de edad, los porcentajes fueron Estados Unidos $58 \%$ y corea del Norte $20 \%$ (Kim, 2006: 284).

Orra encuesta del mismo año (JoongAng Itbo, octubre de 2004$)$ ofrecio una visión positiva de los estadounidenses $(65 \%)$, desfavorable respecto del presidente Bush (72\%), negativa respecto de

Fucente: hetp://www.chrorg/pullication/ $1.359 .3 \%$ 
la invasion de lrak $\left(85^{\circ} \%\right)$ y favorable al candidato demócrata kerry $(68 \%)$ (Kim, 2006: 284).

En las entrevistas realizadas en Seúl, los expertos mencionaron el sentimiento anti-estadounidense, aunque tuvieron opiniones bastante variadas respecto del rol de Fstados Unidos. Algunos expresaron su interés en la unificación, pero otros se refirieron al interés estadounidense por mantener el statu quo. Desde otro punto de vista, Fstados Unidos estaria de acuerdocon la unificación bajo la modalidad de absorción. Por otra parte, otros expertos fueron de opinión de que después de la unificación Estados Unidos debería retirar sus tropas de la península. También manifestaron claramente la oposición de este país respecto de la ayuda surcoreana a Corea del Norte. Por último, para alyunos el apoyo estadounidense era necesario, pero dependería de si el gobiemo era republicano o demócrata.

\subsection{El rol de China: ¿por qué es tim active?}

La relación entre China y Corea es tan antigua como la propia existencia de este ultimo y solo se vio debilitada desde el siglo XIX. Los chinos percibicron la guerra de Corea como un acto de agresión en su contra (Kim, 2006: 48). I a identidad socialista china (diferente de aquella de la (Unión Soviética) se desarrolla a partir de dicha guerra (Kim, 2006: 50).
El armisticio con Estados Unidos fue el primer éxito diplomático chino, puesto que llevó a cse país a la mesa de negociaciones. Cabe recordar que la guerra tuvo grandes repercusiones en China. Fn efecto, le significó 740.000 muertos, incluido un hijo de Mao. Por otra parte, debido a los costos de la guerra, perdio la posibilidad de recuperar Taiwán por la fuerza (Kim, 2006: 51).

Ia agudiación del conflicto sinosoviético a partir de los años sesenta, auncue surgido en el decenio anterior con las críticas de Kruschev a Stalim, le dio mayor margen de maniobra a Kim Il-sung a partir de un equilibrio con ambos actores comunistas. Las relaciones sino-coreanas se consolidaron en 1953 y en ese decenio se fortalecieron mediante la cooperación económica (200 millones de dólares), cultural y militar de largo plazo. La presencia militar china se mantuvo hasta 1957 y contribuyo a la reconstrucción de la intraestructura y el ejército del país (Kim, 2006: 51 ).

China concibe a Corea del Norte como un Estado tapón, ya que históricamente muchas de las amenazas de que ha sido objeto se han originado alli, por ejemplo las de Japón y Estados Unidos. En virtud del Tratado de 1961, estableció una reliación "especial" con Corea.

Luego de que Deng Xiaoping llegara al poder en China a fines de los años setenta, la política exterior comenzó a desideologizarse a partir de un concepto de "linea de política exterior independiente" (Kim, 2006: 51). Fn 1992 se firmó el comunicado conjunto de 
mutuo reconocimiento con Corca del Sur (Kim, 2006: 52), ya cue el modelo de Estado desarrollista de este país era visto con simpatía por China y una mejora de las relaciones podía ayudar a su desarrollo económico.

L. . Nordpolitik (1988) de Corea del Sur respecto de China tuvo algunas fases de acercamiento previo, especialmente a principios de los años ochenta con el tema de Taiwán, que se profundizó a fines de esa década con motivo de los Juegos Olímpicos. Cabe recordar que Corea del Sur no condenó el episodio de Tiananmen y que incluso aumentó sus vínculos con China, por ejemplo, en materia de turismo. Por otra parte, hubo una cooperación sustantiva en los organismos multilaterales.

Un antecedente importante de la relación entre China y ambas Coreas es que en 1980 su comercio con Corea del Norte alcanzó los 677 millones de dólares (con una balanza comercial favorable a China de 71 millones) y con Corea del Sur los 188 millones de dólares (y una balanza comercial negativa de 42 millones). En 2004, el comercio de China con Corea del Norte se elevó a 1.384 millones de dólares (con una balanza comercial positiva de 214 millones) y con Corea del Sur a los 79.348 millones de dólares (y una balanza comercial de 20.178 millones) (Kim, 2006: 56). Esto refleja muy claramente la tendencia de las relaciones con ambos países. Se prevé que en 2012 el comercio bilateral entre China y Corea del Sur alcanzará los 200 mil millones de dólares (Kim, 2006: 56).
China estableció relaciones diplomáticas con Corea del Sur en 1992 y mantuvo una política orientada a evitar un mayor involucramiento en el conflicto coreano. Cabe recordar que uno de los criterios de política exterior de China era la noción de "maxi-mini", esto es, de maximizar los derechos e intereses y minimizar los costos y responsabilidades. Solo en 2003 lanzó una agresiva diplomacia de mediación. El problema no era el programa nuclear coreano en sí, sino principalmente el conflicto potencial entre Estados Unidos y Corea del Norte en su patio trasero estratégico (Kim, 2006: 61).

Entre las causas que condujeron a un mayor involucramiento chino en el conflicto de la península se contó la preocupación que despertaba en China y Corea del Norte la doctrina Bush en Irak. Por otra parte, el lenguaje agresivo de "Estado paria" y de "eje dol mal" de la política exterior estadounidense podía generar una situación muy peligrosa. De acuerdo con la postura nuclear norteamericana de 2002, China y Corea del Norte eran dos de sus siete objetivos principales, lo que contradecía las garantías que Estados Unidos le ofrecía a Corea del Norte. Además, el primero había redefinido su doctrina nuclear buscando crear armas nuevas, pequeñas y "usables". Por otra parte, Estados Unidos procuró involucrar a China en el aislamiento de Corea del Norte.

La política china hacia Corea del Norte incluye "los cinco no": no armas nucleares, no refugiados, no colapso, 
no inestabilidad y no guerra. China juega un rol proactivo en el manejo de este conflicto, rechazando las sanciones y entendiendo que ellas no son el factor dominante. La prioridad es la "no guerra" (Kim, 2006: 66).

Para China, la península de Corea es un escudo estratégico vital. Fl peligro de una posible escalada es más alta que en otros conflictos. La frontera sinocoreana, que es de $1.360 \mathrm{Km}$., sirvió de punto de partida a las fuerzas imperiales japonesas en la década de los años treinta y a la aproximación norteamericana de los años cincuenta, que fue percibida como un posible ataque. Además, en la frontera se unen cuatro de las mayores potencias mundiales -China, Japón, Rusia y Estados Unidos-, entendiendo el Océano Pacífico como un lago norteamericano (Kim, 2006: 43).

China es el único país que mantiene relaciones plenas y multidimensionales con ambas Coreas. La opinión pública de Corea del Sur considera que China es un socio. Sus elites tienen una imagen positiva de este país debido a su desarrollo económico (es su principal socio comercial y destino de la inversión externa directa), al eventual apoyo que prestaría a las políticas de reconciliación intercoreanas y a que representa una posibilidad de reducir su dependencia de Estados Unidos. Por otra parte, la política hacia ambas Coreas buscaria mantener el statu quo. Fn sus relaciones con la península, China procuraría "buscar intereses compartidos preservando las diferencias" (Qiutong cunyi) (Kim, 2006: 69).
Entre los objetivos estratégicos chinos en relación con la península coreana, cabe mencionar los siguientes: mantener la paz y la estabilidad en la península, promover el intercambio económico y la cooperación, contribuir a la supervivencia del régimen norcoreano, prevenir la dominación de Corea por un poder externo, detener el flujo de refugiados norcoreanos y de misioneros cristianos surcoreanos en la provincia de Jilin, trenar el etmonacionalismo entre los chino-coreanos, prevenir la formación de una coalición anti-China en Asia y manejar cuidadosamente la relación triangular con ambas Coreas (Kim, 2006: 63).

Como principales obstáculos para alcanzar estos objetivos se cuentan la conducta de seguridad (o inseguridad) de Pyongyang (programa nuclear y de misiles); la crisis de 1993-1994 entre Corea y Estados Unidos; la crisis de los misiles Taepong, de 1998-1999; la línea dura de George W. Bush y la reciente crisis nuclear (Kim, 2006: 63).

Cabe recordar que la crisis de 19931994 dificultó una relación triangular equilibrada de China con las dos Coreas y que en ese cntonces amenazó con utilizar el veto. La crisis fue un éxito diplomático para Corea del Norte, porque condujo a un diálogo bilateral con Estados Unidos, pero significó que China jugara un papel secundario afectado por Tiananmen y la crisis del comunismo ( $\mathrm{Kim}, 2006$ : 64-65). Decidió no unirse al $\mathrm{KEDO}$ debido a la lógica del sistema maxi-mini, pero durante la crisis nuclear de 2003 desa- 
rrolló una diplomacia preventiva. ¿Por qué motivo?

La principal razón que tuvo China para acrecentar su nivel de acercamiento hacia Corea del Norte fue el peligro de que se produjera un ataque militar de Estados Unidos. I a presión de este podía inducir a Corea del Norte a atacar primero, anticipándose a una medida preventiva estadounidense, curso de acción que podía considerarse racional, aunque la victoria fuera imposible. En 2003, Bush había enviado claras señales militares cuando ordeno desplazar al portaaviones Carl Vinson y luego envió 12 B- 1 y 12 B-52 a Guam (Kim, 2006: 66). Una solución militar impuesta por Washington podía constituir una victoria geoestratégica muy importante, a expensas de China.

China ha desempeñado un papel extremadamente intenso como "mediador activo" en las "conversaciones de las seis partes". En 2005, como medida para defenderse de Estados Unidos, Corea anunció que poseía armas nucleares, lo que también significaba suspender en forma indefinida su participación en ese diálogo. Ante esto, China envió diplomáticos a Pyongyang y sus presiones, junto con una adeculada coordinación con Corea del Sur, llevaron a los norcoreanos a negociar nuevamente para llegar a una cuarta ronda de "conversaciones de las seis partes». El objetivo inicial de Corea del Norte era establecer un diálogo bilateral con Estados Unidos y la derivación hacia su posterior incorporación en las "conversaciones de las seis partes" fue un éxito diplomático chino (Kim, 2006: 68).

El Tratado de Amistad, Cooperación y Asistencia Mutua, de 1961, que establecía "relaciones tan estrechas como labios y dientes", fue pieza fundamental de las relaciones entrc China y Corea del Norte. Sin embargo, este tratado es solo simbólico y está claramente obsoleto. Desde la guerra de Corea, y sobre todo desde la muerte de Kim Il-sung en 1994, los contactos entre el Ejército Popular de Corea y el Ejército Popular de Liberación (EPL) chino han declinado en frecuencia, nivel y sustancia. Las actuales relaciones militares parecen ser más simbólicas que sustantivas y muchas visitas del EPL son descritas como de "buena voluntad". Con Corea del Sur se han realizado numerosas cumbres, micntras que en el caso de Corea del Norte solo en el año 2000 se realizó una reunión entre Kim Jong-il y Jiang Zemin (Kim, 2006: 71).

A juicio de China, las alianzas militares o multilaterales son reliquias de lia guerra fría. Extraoficialmente, se ha dicho que la alianza entre China y Corea del Norte no entraña el compromiso de defender al segundo, que no respaldará un ataque no provocado por parte de Corea del Norte o que no intervendría "automáticamente" (Kim, 2006: 72). Por otra parte, en el discurso de defensa de Corea del Sur se evita poner a China como una amenaza, producto de lo cual en 1999 decidió no participar en el sistema de defensa anti-misiles de los Fstados Unidos (Kim, 2006: 74). 
En marzo de 2005, el presidente Roh advirtió que podría no estar del lado de Estados Unidos y Japón en un eventual conflicto contra China y Corea del Norte. Su visión al respecto ha estado más cerca de China que de Estados Unidos (Kim, 2006: 74-75). Como se dijo, Corea del Sur y China pretenden alcanzar un comercio de 200 mil millones de dólares en 2012 (Cumbre de noviembre de 2005 entre Hu y Roh).

Fn las cutrevistas realizadas en Seúl, los expertos surcoreanos destacaron el interés chino por mantener el statu quo (o, al menos, por un proceso lento de unificación). Otros expresaron que China podría estar de acuerdo con la unificación, pero siempre que desempenara un papel importante en el proceso. También señalaron que este país es el unico que influye en Corea del Norte y recordaron que durante una crisis anterior ejercieron presión sobre este último, interrumpiendo el flujo de recursos energéticos para forzar algunas reacciones. Corea del Norte necesitaría el apoyo económico chino y la percepción general es que este le daría gran importancia a China, para locual se requeriría el "consenso de Beijing" además del "consenso de Washington". También se destacó que China no quiere que haya tropas estadounidenses en la península. Finalmente, algunos expertos consideran que China es mejor amigo de Corea del Sur que de los Fstados Unidos o Japón.
3.3 ¿Le conviene a Japón una Corea unificada?

Desde la perspectiva japonesa, Corea ticne importancia fundamental para su política exterior. Ios contactos históricos a que dio lugar la proximidad geográfica entre ambos tuvieron gradualmente connotaciones negativas debido a que durante siglos Japón ha mantenido fuertes intereses geopolíticos en la península. Por una parte, los intentos mongoles de invadir Japón se realizaron a través de la península coreana y, por otra, tras el predominio de Toyotomi Hideyoshi sobre sus rivales en el siglo XVI, Corea se convirtió en un objetivo permanente para la expansión de Japón (Kawashima, 2003: 74).

Tan pronto como se inició la restauración Meiji en Japón, en 1868, el país comenzó a intervenir en la península coreana (Kawashima, 2003: 75). Las victorias japonesas sobre China en 1894-1895 y luego sobre Rusia en la guerra sino-japonesa de 1904-1905 precipitaron la hegemonía nipona en la península (Green, 2003: 113). Para el nacionalismo japonés, esta representaba «un dardo apuntado al corazón de Japón" (Kim, 2006: 168) y, posteriormente, una oportunidad de desarrollo por ejemplo, mediante el establecimiento en Corea de conglomerados industriales o "zaibatsus" (Kim, 2006: 170). Hasta el término de la ocupación, en 1945, Corea se consideraba fuente de materias primas, trabajo y energía.

A los factores históricos se sumó una constante intervención japonesa 
que aún tiene importancia significativa en las relaciones entre ambos paises y que, junto con la ocupación japonesa desde 1910 hasta 194.5, son elementos extremadamente negativos.

Respecto de este ultimo periodo, hay temas pendientes y algunas disputas limitrofes. Unos de ellos es la soberania sobre las islas Tok Do (Takeshima en japonés), ocupadas por la policía coreana desde 1953 (Kim, 2006: 169). Otro es el de las esclavas sexuales coreanas durante la Segunda Guerra Mundial, cuyo número fluctúa entre 100 mil y 300 mil (Kim, 2006: 170). A ello se suma la migración obligatoria de coreanos a Japón (Zainichi) y su conformacion como ciudadanos de segunda clase (Kim, 2006: 170) y, más recientemente, el tema de los textos relativos a la Segunda Cinerra Mundial y de las visitas al templo de Yasukuni, que comenzaron a mediados de les años ochentia (Kim, 2006: 17.3-174). Todos ellos han afectado las relaciones en forma significativa.

Cabe recordar que en Asia oriental el sistema estadounidense de alianzas bilaterales (que se daba en términos de "hub and spoke") no daba resultados (Kim, 2006: 171). En este contexto, en los anos cincuenta Japón buscó regularizar sus relaciones vecinales con Taiwán, Birmania, Filipinas, Indonesia y Viernam de Sur. L a normalizacion de estas con Corea del Sur y Malasia solo legaría en la década de los sesenta. la repatriación de norcoreanos desde Japón en los años cincuenta estimuló ell alguna medida el proceso. Las rela- ciones entre Japón y Corea del Sur se restablecieron durante la dictadura de Park Chung-hee, pese al fuerte sentimiento antijaponés en este último país (Kawashima, 200.3: 76).

El diálogo intercoreano de fires de los años ochenta y la Nordpolitik del presidente Roh Tike-woo estimularon un acercamiento japonés (y estadounidense) hacia Corea del Norte. Este buscaba recursos para su desarrollo y vio una buena oportunidad en el acercamiento con Japón. A lo anterior se sumaron las demandas niponas de que les entregaran a los secuestradores aéreos refugiados en Corea del Norte, a las que este respondió exigiendo que primero se normalizaran las relaciones. Japón incluyó luego el tema nuclear, la reanudación del diálogo intercoreano, la admisión como miembro de las $\mathrm{Na}$ ciones Unidas y un tema particularmente dificil, el primer caso de japoneses secuestrados por Corea del Norte desde los años setenta.

En los años noventa hubo varias rondas de conversaciones (ocho, entre 1991 y 1992). Corea del Norte exigía disculpas, compensaciones y que se declarara la mulidad de los tratados que se le habían impuesto históricamente. Japón planteó que no habría compensaciones, que el acercimiento no afectaria las relaciones entre Japón y Corea del Sur, las deudas coreanas, el estatus de las parejas binacionales y otros.

l.uego de la impresión que causaron en Japón las pruebas misilisticas norcorcanas a fines de los anos noventa. que afectaron profundanente las po- 
sibilidades de reunificacion, en 2000 se reanudaron las conversaciones. A los temas anteriores se sumaron los de "cooperación economica" pensación" desde el punto de vista de (orea del Norte) y las donaciones de alimentos, y se retomó el examen del toma de los secuestros (al aumentar cl numero casos). Se pidio detener el programa de armas de destrucción masiva y el programa nuclear de Corea del Norte, lo que este consideró inaceptable Norte y lo llevó a suspender las conversaciones ese mismo año.

El diálogo se reanudo en 2002 y se avanzó en lo relativo a los secuestros y el tema muclear. Japón señaló que no habria normalización a menos que se solucionaran los temas citados -secuestros, armas y programa nuclear-. En 2004 continuaron las conversaciones sobre las mismas materias y Japón prometió no imponer sanciones económicas.

Ante la interrogante de si a Japón le convienc la unificación de Corea, parte de la respuesta radica en que busca unia Corea unificata que sea amistosa a la ver con Japón y los Fstados Unidos, comomicamente viable y políticamente abierta y yue permita la continuidad de la presencia estadounidense (Arnacost y Pỵle, 2003: 126). Si el parís unificado conserva armas nucleares, se inclina hacia China, se rehúsa a continuar la relacion con los Fstados Unidos en materia de seguridad, admitiendo una presencia militar residual, o bien es hostil a Japón en su visión de futuro, seria una derrota para la polírica exterior de este país y una eventual amenaza (Armacost y lyyle, 2003: 126). Desde el punto de vista económico, la unificación tampoco parecería del todo descable, dado que ambos países compiren en un sinnumero de sectores, aunque han desarrollado una creciente interdependencia (Armacost y Pyle, 2003: 126).

En las entrevistas realizadas en Seúl, quedó de manifiesto que a Japón se le atribuye un rol secundario. Ios entrevistados descarían un cambio de régimen en la península coreana. Para algunos, Japón constituye un obstáculo para la unificación o no tiene interés en ella y una Corea unificada podría ser preocupante como posible competidor. Pese a cllo, uno de los entrevistados señaló que a partir de la unificación Japón podría obtener algunos beneficios económicos. Por otra parte, algunos expresaron una visión más radical respecto del papel de Japón y le atribuyeron la verdadera responsabilidad de la división de la península.

\section{4 ¿Desempeña Rusia algúm papel importante?}

I a península coreana ocupa un lugar secundario en la política exterior rusa hacia el noreste asiático, que se centra en Fstados Unidos, China y Japón, de manera que las relaciones rusas con la península dependen de las entahladas con estos países. Históricamente, sus intereses en ella han sido de indole más bien estratégica que económica. Rusia solo llegó a compartir una frontera con 
Corea cuando anexó la provincia marítima descle (hina mediante el tratado de Pekín, en 1860) (Kim, 2006: 110$)$.

Por otra parte, el tema de las relaciones entre Rusia y la peninsula coreana se maneja a nivel de la burocracia de política exterior, y no de los funcionarios de alta jerarquia, por lo cual es incremental y lenta. Al respecto, las excepciones han sido ciorbacher en 1989-1990 y la politica de Putin en el período 2000-2002.

El pensamiento geoestratégico de Rusia se divide entre "europeistas", "asianistas" y "excepcionalistas" (el) línico pais realmente eurasiáticol y está significativamente relacionado con su búsqueda de identidad. Por otra parte, su deseo de recuperar poder e influencia $y$ de volver a ser un actor relevante a nivel mundial y regional influye marcadamente en sus politicas hacia el Asia oriential.

Las relaciones entre Rusia ya ambas Coreas están fuertemente marcadas por su pasado (zarista, comunistay poscomunistal y sus vinculaciones con los distintos reinos o Estades del territorio coreano (Choson, la Corea colonial y las dos Corcas actuales). Ya a mediados del siglo XIX. Francia, entre otros, miraba con recelo las intenciones rusas en la península, én circunstancias de que la influencia rusa disminuyou tras la derrota ante Japón a principios del siglo XX (Kim, 2006: 110-111).

En sus incios, la Union Soviéticia tenía muchos inmigrantes coreanos y chinos. Stalin deportó a I 80 mil coreanos al Asia Central, ante cl temor paranoico de que se comvirtieran en espias japoneses. I.os inmigrantes fueron obligados al traslacto, a realizar trabajos forcados y cnviados a prisión. Fn los años treinta fallecieron 20 mil de ellos dehido a las condiciones a que eran sometidos. También fueron enviados a las islas Kuriles (Kim, 2006: 111).

I.os soviéticos cligieron a Kim IIsung. primer lider de Corea del Norte, comosu interlocutor válido en ese país debido a sus cualidades particulares de liderazgo. En ese período habia en Seúl grupos nacionalistas, communistas retornados descle (hina (Yan'an), soviético-coreanos (que formaban parte del ejército soviético) y partisanos (Kapsan. grupo liderado por el propio Kims.

la búsqueda de un equilibrio entre China y la Unión Soviética por Kim llsung mediante su política de "Juche" dio lugar a una relación triangular que no fue estable. Fn efecto, Corea del Norte se distanció de la Unión Soviética en los años sesenta, con el periodo de $\mathrm{Ni}$ kita Khrushchev. I.uego se produjo un acercamiento en el periodo de Brezhnes, que concibió a Corea del Norte como un aliado estratégico.

En les anos ochenta la Union Soviética intertó recomponer sus relaciones con Corea del Norte, en parte a raíz del acercamiento sino-estadounidense. Además, Konstantin Chernenko le proporcionó apoyo técnico energético -tanto convencional como nuclear-y nuevo equipamiento militar.

Corbachev continuó el accramiento (operaciones navales conjuntas, uso de puertos coreanos y otros), perocon 
la variante de una politica hacia ambas Coreans, cuando decidió participar en los Juegos (Olímpicos y acercarse a Corea del Sur. A partir de ello se restablecieron las relaciones consulares, se abrieron oficinas en ambos países, se establecieron vínculos marítimos (Vladivostok-Pusan) y los soviéticos se refirieron puiblicamente en términos positivos a los éxitos económicos de ese país. Todo ello condujo a un enfriamiento de sus relaciones con Corea del Xorte. Cahe recordar que la Perestroika se inició en 1985 y que la Norpolitik del presidente Roh Tae-woo comenzo en 1988 (Kim, 2006: 119-120).

Si bien la Unión Soviética habia planteado su apoyo a Corea del Norte y se había pronunciado por una Corea desnuclearizada, en la ćpoca de Corhachev la situación de la península aparecia como el principal obstáculo para establecer un sistema de seguridad asiático (Kim, 2006: 118).

Gorbacher y Roh se reunieron en San Francisco, en 1990. Conversaron sobre la posibilidad de normalizar sus relaciones, lo que fue informado a Corea del Norte. Este señaló que ello cra "desagradable, nauseabundo e indecoroso" y yue la Union Soviética tenia una "diplomacia comprada con dólitres". Incluso amenazó con apoyar las reclamaciones japoneses sobre las islas Buriles, que estaban bajo el control de la Unión Sovićtica (Kim, 2006: 120).

Fn una nueva reunión realizada en Cheju, Corea del Sur, en 1991, (iorbacher anuncio el apoyo al ingreso de Corea del Norte en las Niaciones Unidas para desarrollar un diálogo intercoreano orientado a reducir las tensiones: supervisar el desarrollo nuclear de ese país (Kim, 2006: 121).

Tras el término de la Unión Soviética, durante el periodo de Yeltsin las relaciones fucron extremadamente frías. En Rusia predominaba un anticomunismo que mirala con muy malos ojos el modelo coreano, como resabio stalinista; por otra parte, buscaba acercarse a Corea del Sur a fin de recabar apoyo para su desarrollo.

Yeltsin decidió no renovar el Tratado de Mutua Defensa y Cooperación, de 1961, suscrito entre la Unión Soviética y Corea del Norte (Kim, 2006: 121); Corea del Sur queria que renunciara a sus cláusulas militares. Fn 1992 se firmó el Tratado sobre Principios de Relaciones entre la Federación de Rusia y la República de Corea, sobre las bases de libertad, democracia, derechos humanos y economia de mercado. Además, suspendió la ayuda militar a Corea del Norte. Por su parte, este consideraba que el trat tado era obsoleto y nocreía en las garantias de que el paraguas nuclear ruso efectivamente lo incluía, y redefinió su política de seguridad. También señalo yue habia sido "traicionadoy dañado en un sentido material" (Kim, 2006: 124).

Rusia quería desempeñar un papel relevante en el escenario internacional, pero de lo dejó de lado en el proceso de solución del prohlema nuclear de la peninsula coreana (las conversaciones de las cuatro partes incluyeron a China, perono a Rusia). Por otra parte, Corea 
del Norte no tenía especial interés enl la participación rusa y locomminaba a pedir el retiro de los Estados Unidos de la península y a interrumpir el despliegue del sistema estadounidense de defensal de misiles's en el país.

Cabe destacar que en los años noventa los rusos no creian que Corea del Vorte estuviera deteniendo su programa nuclear ni que dicho programa afectara directamente su seguridad, punto que no cra compartido por los militares.

Bajo la administración del presidente Putin, en 2001 se buscó nuevamente aprovechar las distancias entre Kim Dae-jung, Presidente de Corea del Sur, y los Fistados Unidos, de manera que se inició un acercamiento con Corea del Sur en materias de seguridad.

Por otra parte, Rusia firmó un Tratado de Amistad, Buena Vecindad y Cooperación con Corea del Norte (Kim, 2006: 127). Fste no incluyó la intervención militar automática, pero si una ambigua cláasula de "contacto mutuo" en caso de crisis. Tras una visita de Putin a ese país en 2000, se normalizaron las relaciones. P'utin aprobó la venta de repuestos para el armamento norcoreano y de algunos equipos, pero no de armamento avanzado; además, se generaron ofertas de cooperación energéticia.

Moscí sigue considerando a Corea del Norte como un Fistado tapón (bu-

Inicialmente, el Sistema de lefensa de Misiles de Teatro (TMLI) / estadomidense, peroluege alosorbido en el Sistema Nacional de lectensa de Mlisiles (NI)N1). ffer) en sus relaciones regionales. En gencral, la politica rusa hacia este país puede calificarse como pasiva, reactiva y bastante cautia. Cabe recordar la importancia de aspectos tales como el debilitamiento del Lejano Oriente ruso, de repercusiones geopoliticas, el pesimismo ruso respecto de las "conversaciones de las seis partes"y su preocupación por la agresividad estadounidense hacia Corea del Norte (Kim, 2006: 133).

F.l tema energético cruza ambas Cortas, que son altamente dependientes de los recursos energéticos externos, sobre todo Corea del Sur. En Corea del Norte hay una escisez de recursos tales como el carbón y los recursos hidricos! carece de reservas de petroleo. I a alternativa es importar recursos cnergéticos continentales descle China y Rusia ! produce energía mediante instalaciones nucleares anticuadias. Fl desirrollo de tecnologías menos riesgosas con apoyo internacional (KFI)()) está suspendido. Aplica un sistema de racionamiento de encrgia.

Por otra parte, Corea del Sur importa casi la totalidad de la energía que consume (la mayor parte del petróleo proviene del Medio Orientel, prexura diversificar sus importacione's de gas y perróleo (por ejemplo, descle Rusia) y 
se prevé que también incremente cl uso de plantas nucleares.

I a posible obtención de recursos energéticos de Rusia ha influido positivamente en la relación entre ambos países. Por otra parte, China ha utilizado las conexiones energéticas con Coreil del Norte para presionarla a sentarse en la mesa de negociaciones durante recientes periodos de crisis. Se ha estudiado una eventual conexión energética desde Rusia, vía China y Corea del Norte hacia Corea del Sur, aunque estratégicamente parece inviable (Kim, 2006: 152).

En las entrevistas realizadas en Seúl, los expertos mencionaron a Rusia como ef actor más secundario, lo que algunos atribuyerom al papel ambivalente y carente de interes real por la unificación de la península coreana. Al respecto, se pronunciaron a favor del statu quo.

\section{Conclusiones}

En general, y a partir de lo yue hemos visto, puede concluirse que desde el témino de la guerra fría se ha producido una variación importante en las alianzas regionales. Fn esa épocia, Corea de Vorte procuraba mantener cicrta cercania con la Unión Soviética y con la República Popular China mediante la "Juche", pero dentro de

lenergy hoformation Administration, ofticial Forergy Statistics from the U.S. (iosernment, hetp://wwweda.dot.gow/cabs/ South_Kore:a/Backgroumd.homl y luttp:// wwwida.doe.gov/cahs/North_Koread Background.html. cierto equilibrio que le impidiera caer dentro de la órbita de uno de ellos. Cabe recordar que hasta comienzos de los años setenta Corea del Norte era economicamente más desarrollado que Corea del Sur. Tras la llegada de Gorbacher y la política hacia ambas Coreas, (hina siguio una política similar como parte de sus intentos por desideologizar su política exterior. En este contexto, encontramos una Corea del Norte cirda vez más aislada, cuyos viejos aliados buscaban una política de compromisos e integración económica con su rival del sur. Por otra parte, Corea del Sur, estrecha aliada de los Estados Unidos durante las dictaduras militares, comenzó a acercarse más a otros actores tales como China.

Yi desde la Nordpolitik del presidente Roh Tac-woo, que apuntaba a mejorar las relaciones con los países socialistas, Corea del Sur comenzó a integrarse de forma más sustantiva con China. Como se he visto, este pais fortaleció los lazoos con Corea del Sur al punto que resulta dificil apreciar el alcance $y$ profundidad de una asociación estratégica futura. Las relaciones entre China y Corea del Norte son solo formales. Rusia y Japón no tienen demasiada injerencia en el eventual futuro de la península coreana y su unificación nacional. Mientras que a Japón le cuesta visualizar una Corea unificada, a los coreanos les resulta dificil concebir a Japón desempeñando un rol sustantivo en su unificación. Cuando Rusia logre superar su crisis de identidad, tal vez pueda avanzar más allá de los vínculos energéticos, auncque 
estos son un buen primer paso hacia una mayor relevancia.

En sintesis, nos encontramos ante una Corea del Norte aislada y una Corea del Sur que transita estratégicamente desde una alianza con los Fstados Unidos hacia una con China, auncue en alguna medicla esto puede invertirse por la nueva administración surcoreana, de manera que no es posible apreciar claramente su grado de coherencia.

Percibimos que las políticas de unificación nacional de Corea del Sur, los esfuerzos del regimen norcoreano por sobrevivir y los intereses estratégicos de Estados Unidos, China, Rusia y Japón en la península pueden ser contradictorios en algunos niveles.

Las políticas de unificación nacional han perdido algo de su energía inicial debido a la mayor politización del tema por los actores políticos. Por otra parte, en Corea del Sur la percepción de los sectores académicos proclives tanto al gobierno como a la oposición prevén que la unificación tardará entre 20 y 40 anos. Al parecer noconvendría una unificación por colapso, debido a los costos conómicos que esta tracría consigo.

Está claro que los actores externos relevantes en el nuevo escenario de las politicas de unificación nacionat son China y Estados Unidos. Fl primero ha logrado reconfigurar su relación con la península a un grado sorprendente e inesperado y és el único que mantiene una política coherente hacia ambas Coreals. Fstados Unidos, por su parte, ha tratado de forzar a Corea del Norte a poner fin al programa nuclear $y$ a los programas de armas de destruccion masiva, pero ha caidoren un discurso demasiado agresivo que no solo afecta sus relaciones con la península coreana, sino con otros actores de la región. Sin embargo, más allá de la retórica de la administración del presidente Bush, nos encontramos con una política cue a nuestro juicio comienza a ser más coherente y, evidentemente, más responsable que en otras regiones.

Si bien China y listados Unides, los principales actores, scrían eventuiales facilitadores del proceso de unificación de la peninsula coreana, no lo son al punto de desear una unificación que altere demasiado los equilibrios estratégicos regionales. En otras palabras, podrían facilitar el proceso, pero actualmente no constituyen un respaldo sólido a la ansiada unificación coreana. Es probable que esta dependa más de la voluntad de los propios coreanos, o de una crisis inesperada en Corea del Norte que los obligue a jugar algunas cartas que a primera vista resultan muy duras de asumir. Sin embargo, creemos que si algo sobra en Corea es fortaleza y voluntad para superar las vicisitudes. Ante la interrogiante de si el sistema de alianzas regionales favorece un proceso de unificación nacional de Corea, habria que responder con escepticismo que tal vez no lo hace.

\section{Bibliografía}

Armstrong, (harles K. (2004), "Inter-kiorean relations: A North Korean lerepective" (') Kim, Samucl, Inter-Korean Relations. Problens and Prospects, New York, Palgrave Macilillaw, p. 41 . 
Armacost, Mlichael H. I Kenneth B. Pyle 1200.31. "Japan and the Enitication of Korea: (hallenges for II.S. Policy Coordination" en Nicholas fherstadt y Richard I. Ellings, Koreats Future and the Great Ponerers, Seattle: Eniversity of Washingtom P'ress, p. 126.

Bechtol Jr., Brose F., Astessing the Present and (harting the Future of U.S.-LP'RK Relations: The Political-Diplomatic I)imension of the Vuclear Confrontation, con kwack, Tate-hwan y Jow, heung-holed. $)$. 2006, The linited State's and the Korean

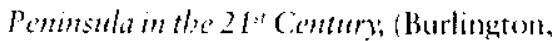
Asligite, P. IJ6.

(astillo, Sergio (20)2), Ia untication de Comea. It cpilego de la genera fria, Madrid: Fiditorial Cataratil, p. If6.

Cumings, Bruce (2004), Fll lugar de Coreden al sol, cirdohat Fditorial commicarte, P?. $243-349$.

(ha. Victor I). Y David (.. King (2003). Vuclew Vurb Korea. A Debate on tngagement Strategies, New York: Columbia University P'ress, pp. 140-144.

Herestadt, Nichohas (20)(34), "North Koreats Survival (iame: Inderstanding the Recent l'ast, Thinking about the luture", en Ahn Choong-yong, Nicholiss Fherstadt y Lee Young-sun (ads.), A Now Internationmal Ingagement framearom for North Kored Contendeng Perspectures. W'ashingtom I). C.., Korea Fonomic Institute of Americia, p. 6.5.

(ireco, Michald J. (2003), Jat)ans Reluctamt Kealism, Nueva York: P'algrave, p. II.3.

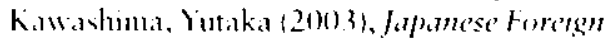
Policy at the Cirossroads. (Wallonge's and Options for the Tu'enty-first Century, Washingeon I).C., Brookings Institutions I'ress, ple - 4-76.

Kim, 1)ate-jung (1998), "Inatugural addresshy Kim 1)ar-jung, the 15th-term President of the Republic of Korea ", http:/www. unikorea.go.kr/english, consultada of 15 de tedrero de $200 \%$.

Kim, Sammel (2006), The Tiee koreas and the Cireat Pene's, Cambridge, Cambridge liniversiry Press, p. 5()- 2.58 .
Lee, Ki-baik (1984), A Neth History of Koreda. Seul, Ilshokak Publishers, pp. 19-31 i.

lece, Young-sun y Yoon Deok-ryong (201)4), "The Structure of North Korca 's Political Fonomy: Changes and fifects", en Ahn Choong-yong, Nicholas Fherstadt y lee Young-sunn (cds.), it Neu Internatomal Fingagenent Franowerk for North Korea Comtending Perspectio'es, Washington 1).(.., Korea licomomic Institute of Americia, pp. 46-.59.

levin, Norman I). y Han, Yong-sup (20012), Sunshrme in Korea: the South Konsum debate ore'r policies toudard North Korea, <w'ww.rand.orgs, consultada d. $1.5 \mathrm{de}$ Noviembre de 2006.

Manhan, Chinhany Pyonhan 199 it, "Corea" en Nahm, Andrew (.., Introdation to Korean Ihstery and Culture', Hollym Corporation l'ullishers, p. 21.

Noland, Marcus (2004), . Political liconomy of North Korea: Historical Bactiground and l'resent Situation", en Ahn C.hoongyomg, Nicholas Eherstadt y I ee loumg-sum (eds.), A Nen Internatiomal Engage'ment Frame'terek for North Korea Contendmp Perspectines, Washington D.C.., Korea Foomomic Institute of Americil, p. 19-20. Pollack, fontathan 1). y lee, (hung-min (1949), Preparing for Korest linification. Scenaros and Implicatums, Santi Mlenica: Rand (orporation, p. 34-45.

Scalipino, Robert A. (20)(16), . (I.S.-1)PRK Relations in the Kin Jong-il tra "'n Kihl, Youn-whan y Kim. I Iong-nack fods.), Nerth Korea. The Pelitics of Regime Sunn'al. Nueva York: MI.F. Sharpe, Inc. p. $146-152$.

Seth, Michacel J. (2006), A cuncise Hestory of Kurcia, lanham: Rowman \& Littleticlds Publishers Inc.. pp. 16-121.

Wolf, Charlesy Akramm, Kamil (20015), Nomthern Paradexes. Coramstances, forsts, and Comsequences of Korean Linfication, Silnta Mlomica, Rand Corporation, pp. 9.18 . 